\title{
Simulation of Machine Induced Background in the LHCb Experiment: Methodology and Implementation
}

\author{
R. B. Appleby, H. Burkhardt, G. Corti, Y. I. Levinsen, M. H. Lieng and V. Talanov
}

\begin{abstract}
Numerical analyses of machine induced background at the LHC are needed to evaluate the complete running environment of an experiment. In order to have a comprehensive view of the machine background in an experiment all of its sources, ranging from collimators' cleaning inefficiency to distant and local beam-gas interactions need to be estimated; particles showering from the losses are then to be transported all the way to the experimental setup and the response of the detector evaluated. In this paper we describe a novel methodology implemented for the LHCb experiment to achieve this. Each step in the chain is simulated with software specific to the task and provides input to the subsequent step through a well-defined and clear interface. Further, we will discuss in detail the various steps in the chain together with the advantages such a modular method allows in evaluating operational conditions where scaling of the initial sources can be applied. We will also give some examples of the results obtained.
\end{abstract}

\section{INTRODUCTION}

A $\mathrm{N}$ accelerator environment always contains particles not originating from beam collisions at the interaction point (IP). These background particles can reach the detector and give rise to a certain level of background in the LHC experiments. It is important to evaluate the amount and characteristics of this background, not only in the design of an experiment but also during operation in order to minimize its potential impact.

Particles originating from proton beam interactions with the gas residue in the vacuum chamber or with the aperture material of the accelerator on either side of the interaction points and reaching the experimental areas from the machine

The work was supported in part by the Russian Foundation for Basic Research grant (No. 10-07-00435)

R. B. Appleby is with the School of Physics and Astronomy and the Cockcroft Institute, University of Manchester, Manchester, M13 9PL, United Kingdom (telephone: +44-161-275-4223, e-mail: robert.appleby@manchester.ac.uk). He was formally with CERN, CH1211 Genève 23, Switzerland until September 2010.

H. Burkhardt is with CERN, CH-1211 Genève, Switzerland (telephone: +41-22-767-5464, e-mail: Helmut.Burkhardt@cern.ch).

G. Corti is with CERN, CH-1211 Genève, Switzerland (telephone: +41-22767-2376, e-mail: Gloria.Corti@cern.ch).

Y. I. Levinsen is with University of Oslo, Norway and CERN, CH-1211 Genève, Switzerland (telephone: +41-22-767-0989, e-mail: Yngve.Inntjore.Levinsen@cern.ch).

M. H. Lieng was with Experimental Physics 5, Technische Universität Dortmund, Dortmund, Germany (telephone: +49-231-755-4526, email:Magnus.Lieng@cern.ch) until May 2011.

V. Talanov is with Institute for High Energy Physics (IHEP), RU-142 281 Protvino, Moscow Region, Russia (telephone: +7-4967-713-420, email:Vadim.Talanov@ihep.ru).

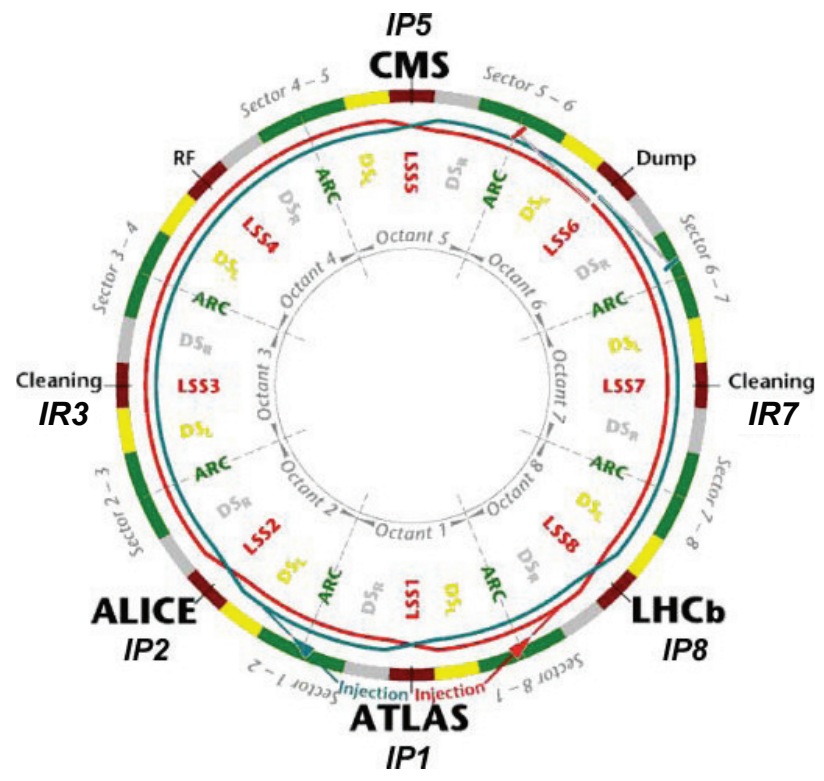

Fig. 1. Layout of the LHC ring. The interaction points are indicated with $\mathrm{LHCb}$ being in IP8.

tunnel constitute what we call the Machine Induced Background (MIB). The rate of this type of background is generally proportional to the machine beam current and depends on a given operating condition; depending on the luminosity of an experiment the MIB may contribute to its radiation environment, influence its trigger and physics measurements.

The Large Hadron Collider (LHC) [1] at CERN started operation at the end of 2009 and, after a short period at 450 $\mathrm{GeV} /$ beam, has been operating at an energy of $3.5 \mathrm{TeV} / \mathrm{beam}$. A planned consolidation will bring LHC to the nominal $7 \mathrm{TeV} /$ beam within the next years. The layout of the machine is shown in Fig. 1. The ring is divided in eight sectors each comprising a long straight section (LSS) of about $600 \mathrm{~m}$ length where the beam trajectory (apart from separation dipoles) is straight. These straight sections are surrounded by dispersion suppression regions (DS) and arcs where the beams are bent in their circular path around the machine by the main dipole magnets. The interaction points where the experiments are located are in the middle of LSSs with the LHCb detector installed at IP8. Insertion regions for betatron and momentum halo cleaning are found in two of the remaining sectors, IR7 and IR3 respectively.

The $\mathrm{LHCb}$ experiment [2] is designed to investigate the 


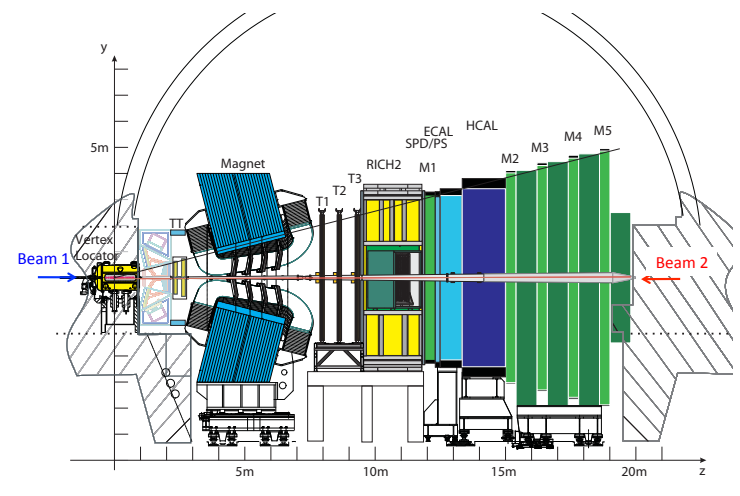

Fig. 2. The $\mathrm{LHCb}$ detector. The directions of the clockwise and counterclockwise beams, respectively beam 1 and beam 2 , are indicated.

possibility of new physics beyond the standard model by performing precision measurements in the beauty and charm sectors. In order to perform such measurements $\mathrm{LHCb}$ intends to operate at an average rate of a few proton-proton interactions per bunch crossing; once the experimental optimal instantaneous luminosity is reached, the pile-up from multiple collisions in single bunch crossings will be optimized by leveling the luminosity profile during the fill. This will result in an increase of the relative MIB to collisions ratio.

The detector, shown in Fig. 2 is a single arm spectrometer in the direction of the clockwise beam (beam 1), and as a result is more sensitive to MIB coming from this beam than the beam going in the counterclockwise direction (beam 2).

\section{Simulation Overview}

A complete chain of simulations has been put in place in order to obtain a comprehensive view of machine induced background in the $\mathrm{LHCb}$ experiment. Dedicated software is used for each aspect of the problem:

- The evaluation of the rates of the different sources of background.

- The simulation of the proton beam interactions with gas or machine aperture restrictions (known as beam losses).

- The transport of the shower to the LHCb cavern.

- The transport of the surviving particles in the detector itself.

Each step in the chain provides the input for the next, allowing to fully evaluate the effect of each background component on the detector, trigger, reconstruction and physics measurement in the experiment. The modular method adopted allows to combine the different sources to provide the overall view of the MIB in a given operational condition. Furthermore, the method permits evaluation of a variety of operational conditions when scaling of the initial sources is applicable, as is the case for MIB contributions directly proportional to local gas pressures.

In the following sections the various steps in the simulation chain are discussed in detail and examples of the results are provided.

\section{SOURCES OF MACHINE INDUCED BACKGROUND}

The total rate of the machine induced background depends on the contribution to the particle flux from secondary cascades originating from the different sources. Secondary particles have different probability to reach $\mathrm{LHCb}$ depending on their origin with respect to the interaction point (IP8). This can be inferred from Fig. 1 where $\mathrm{LHCb}$ is indicated in the LHC layout.

The following MIB sources are relevant for the $\mathrm{LHCb}$ experiment:

1 Inelastic and elastic interactions of the beams with residual gas nuclei in the LSSs close to LHCb.

2 Elastic and diffractive beam-gas interactions in the whole LHC.

3 Betatron cleaning inefficiency at IR7 which results in transverse beam halo.

4 Momentum cleaning inefficiency at IR3, where an offmomentum beam halo is produced.

5 Collisions in the higher luminosity experiments, where a fraction of elastic and diffractive interactions may reach LHCb.

The first source is local to $\mathrm{LHCb}$ and gives a direct background to the experiment. All other sources result in halo buildup where beam protons, due to various processes, are spread in transverse and longitudinal phase space and will impact the aperture restrictions around the machine. As a result some of the MIB sources depend heavily on the LHC machine optics. In the LSS on both sides of LHCb the smallest apertures are given by tertiary collimators (TCTs), designed to protect the quadrupoles triplets and, as a result, the experiment itself. Showers originating from any of the sources hitting the TCTs will give an indirect background to the experiment. While the methodology presented is directly applicable to all MIB sources, in this paper we only consider sources 1, 2 and 3 listed above.

Estimation of proton beam losses are performed individually for the various sources listed above. The first step in the simulation chain for all but the local beam gas source is to evaluate the distribution of the beam losses in the aperture restrictions along the ring and in particular for LHCb losses in the nearby TCT collimators. We consider different machine energies to study the evolution of MIB sources for the possible range of LHC operating parameters.

\section{Distant MIB SOURCES}

Distant machine induced background sources are defined as background components which originate further away than the LSSs close to the interaction point. Therefore the distant MIB sources include the tertiary halo, the elastic or long range beam-gas interactions and the cross talk between the experiments.

The beam will have a limited lifetime, which will generally depend on the beam size (and so emittance), the number of transversal oscillations per turn (tune), alignment and stability of magnet positions, impedance and so forth. As a result, the particles in the beam will drift slowly outwards to higher transversal amplitudes, known as halo growth. This halo 
is removed with a collimation system [3] designed with a three level collimator hierarchy, with the tertiary collimators placed about 70-120 meters away from each interaction point. The proton loss rate on the collimators is estimated by the collimation group [3], [4].

The interaction between the beam and the residual gas in the beam pipe can be elastic or diffractive, in which the proton receives a small angular kick and emerges with only a small energy loss (quasi-zero loss for the case of an elastic interaction). The proton can then stay in the beam for many turns, causing a contribution to the slow halo growth.

Beams collide at the four interaction point in LHC. A fraction of the residues from these collisions will travel with the beam and part of them can impinge on some aperture restriction of another interaction point, producing background to the experiment that is located there. The amount of this depend on the number of collisions in the originating interaction point, the machine optics between the source and the experiment and will never exceed the collision rate. As a result, this background component will become a concern once $\mathrm{LHCb}$ reaches its optimal luminosity while that in the high luminosity interaction points (ATLAS and CMS) will continue to increase. It is expected that the cross-talk from ATLAS will be most relevant for $\mathrm{LHCb}$ as it is in the neighbouring interaction point while CMS is located three octants away.

The tertiary collimators are the smallest aperture restriction close to the interaction points: all the distant background sources produce protons which hit these collimators, resulting in a background flux to each experiment.

There are two main computational issues which are important when studying these long range interactions. The first is the precision in the particle trajectory and aperture model, and the second is the speed of the simulation. Whereas localised shower simulations run over a region of a few hundred metres, these tracking simulations are computing particle dynamics over a few hundred turns of 27 kilometres each. The symplectic six-dimensional tracking code called SixTrack, developed for long term tracking studies at CERN and elsewhere [5], [6] has been used for this work. An extra module was added to SixTrack which allows reading information on the collimators positions and material [7], [8], and to include elastic physics for protons in matter [9]. An alternative approach to the use of SixTrack for the calculation of the LHC halo can be used and is being investigated for future calculations. They are described in [10].

\section{A. Tertiary Halo}

The rate of proton losses on a given collimator has been calculated for nominal optics, collimator layout and settings and a beam energy of $7 \mathrm{TeV}$. The machine parameters used can be found in the LHC design report [11]. An initial transverse halo distribution, either in the vertical or horizontal plane, is tracked for several hundred turns with a complete model of the beam pipe aperture and collimator positions. Re-scattering of protons occurs on the collimator jaws, with elastically interacting protons allowed to continue tracking until they interact inelastically with a collimator jaw or intersect the beam pipe.
The resulting distribution of lost protons around the ring is localized on the primary and secondary collimators in LSS7 but losses are also seen on all tertiary collimators, with a different loss pattern for beam 1 and beam 2 determined by the proximity and the phase advance to the betatron cleaning, and (to a lesser extent, due to less halo build-up) the momentum cleaning section [11] in LSS3. In this paper we only consider the larger losses from betatron beam cleaning. The fraction of total lost protons at a given collimator is termed the cleaning inefficiency, $\eta$. An asymmetry between beam 1 and beam 2 of the losses arises because the tertiary halo is dominantly produced in the betatron cleaning section in LSS7, which is adjacent to IP8 for beam 1 and very far around the LHC ring for beam 2. For this reason we first concentrate on the analysis for beam 1 .

Table I shows the cleaning fractions for the LHC collimation SixTrack calculation of $5 \times 10^{6}$ protons with nominal optics at $7 \mathrm{TeV} /$ beam described in [4] and a $\mathrm{LHCb} \beta^{*}$ of $10 \mathrm{~m}$. The table shows the losses in the horizontal $(\mathrm{H})$ and vertical $(\mathrm{V})$ collimators in LSS8. They are set to $8.3 \sigma$, which is equivalent to $1.35 \mathrm{~mm}$ half-gap for the vertical collimator close to LHCb.

TABLE I

THE PROTON LOSS RATE ON THE HORIZONTAL (H) AND VERTICAL (V) TERTIARY COLLIMATORS CLOSE TO LHCB FOR BEAM 1 FOR A BEAM LIFETIME OF 30 HOURS. THE RATES ARE COMPUTED FOR NOMINAL OPTICS AT 7 TEV BEAM ENERGY AND $\beta^{*}$ OF $10 \mathrm{M}$

\begin{tabular}{ccc}
\hline Halo type & V collimator & H collimator \\
\hline Vertical & $2.57 \times 10^{6} \mathrm{p} / \mathrm{s}$ & $0.10 \times 10^{6} \mathrm{p} / \mathrm{s}$ \\
Horizontal & $7.23 \times 10^{3} \mathrm{p} / \mathrm{s}$ & $0.51 \times 10^{6} \mathrm{p} / \mathrm{s}$ \\
\hline
\end{tabular}

As discussed in the previous section, the halo type refers to the initial halo distribution in the SixTrack calculation, with a pure vertical or horizontal halo referring to the possible extremes of the true halo distribution. The cleaning fractions can be normalized to a proton loss rate through the total beam lifetime. The rate of proton loss on a tertiary collimator is related to the beam lifetime $\tau$ by

$$
\dot{\mathrm{p}}=\frac{\eta}{\tau} \cdot \text { p }_{\text {beam }},
$$

where $p_{\text {beam }}$ is the number of protons in the beam. In this work the betatron halo beam lifetime is assumed to be 30 hours and the total number of protons in the LHC is assumed to be $3 \times 10^{14}$ per beam, giving an initial total loss rate (integrated around the machine) of $2.78 \times 10^{9}$ protons per second. This can be combined to give the proton loss rate at a given collimator, which is shown in Table II for the LHCb tertiary collimators for the cleaning fractions in Table I for beam 1.

TABLE II

THE BEAM 1 LHCB BACKGROUND FLUXES FOR THE TCTS, EXPRESSED IN TERMS OF PER PROTON LOST. THE RATES (PER SECOND) CAN BE OBTAINED USING TABLE I. THE RATES ARE COMPUTED FOR NOMINAL OPTICS AT 7 TEV BEAM ENERGY AND $\beta^{*}$ OF $10 \mathrm{M}$.

\begin{tabular}{ccc}
\hline Collimator & muon rate $[/ \mathrm{p}]$ & charged hadron rate[/p] \\
\hline $\mathrm{H}$ & 0.15 & 0.15 \\
$\mathrm{~V}$ & 0.16 & 0.06 \\
\hline
\end{tabular}




\section{B. Long Range Beam-Gas}

For the studies of the beam-gas component that survives for longer distances, an addition to SixTrack has been developed which reads an ASCII formatted list of proton-gas collision events and distribute them around the ring according to the gas pressure distribution [12], [13]. The beam gas interaction is simulated using the DPMJET-III event generator [14]. The direction and energy of the incoming proton is taken into account and the gas particle taken at rest.

The tracking simulation is usually done over a maximum of 100 turns, with the most significant losses within the first three turns after an interaction took place. The tracking of a proton stops as soon as it has hit the beam pipe or when it has made an inelastic collision with a collimator jaw.

The simulation of long range beam-gas is done with parameters very close to the machine configuration in 2010 (which are not those of the nominal LHC). These parameters are a beam energy of $3.5 \mathrm{TeV}$, no external crossing angle, and a simulated static pressure map for an early unscrubbed machine, with a rescaled dynamic contribution to the pressure in the arcs coming from synchrotron radiation. An average $\mathrm{H}_{2}$ molecule density around the ring of $3.49 \times 10^{11} \mathrm{H}_{2} / \mathrm{m}^{3}$ was used in the simulation [16]. The focussing in LHCb is set to a $\beta^{*}$ of $3 \mathrm{~m}$ instead of $3.5 \mathrm{~m}$, which means that the tertiary collimators are set slightly closer in the simulation compared to how they are set in the machine. In the later part of 2010 the LHC was run with external crossing angle, something that is not considered in the simulation. The difference is not expected to be of large significance to beam-gas studies. The simulated loss maps has to be scaled to a given beam current. With the assumption of 368 bunches/beam and $1.1 \times 10^{11}$ protons/bunch the results obtained are given in Table III.

TABLE III

SIMULATED RATES IN PROTONS PER SECOND HITTING THE HORIZONTAL (H) AND VERTICAL (V) TERTIARY COLLIMATORS AROUND LHCB FOR THE 3.5 TEV/BEAM PARAMETERS.

\begin{tabular}{ccc}
\hline Orientation & \multicolumn{2}{c}{ Rate } \\
& \multicolumn{2}{c}{ [protons/s] } \\
\hline & Beam One & Beam Two \\
$\mathrm{H}$ & 51.9 & 56.2 \\
$\mathrm{~V}$ & 41.5 & 132.6 \\
\hline
\end{tabular}

\section{Output}

All information about the stopping particles are then written to an ASCII file to be used in the shower simulations in the LSSs. This information includes position, energy, and direction of each particle, in addition to the information of the position of the interaction in the ring and what species of gas participated in the beam-gas collision. The latter makes it possible to rescale for pressure changes.

These simulations are simultaneously providing input to all experiments around the ring, in form of loss maps on aperture and collimator hits. It would also be usable for e.g. machine protection studies at other locations. An example of the loss distribution from collisions on hydrogen can be seen in Fig. 3.

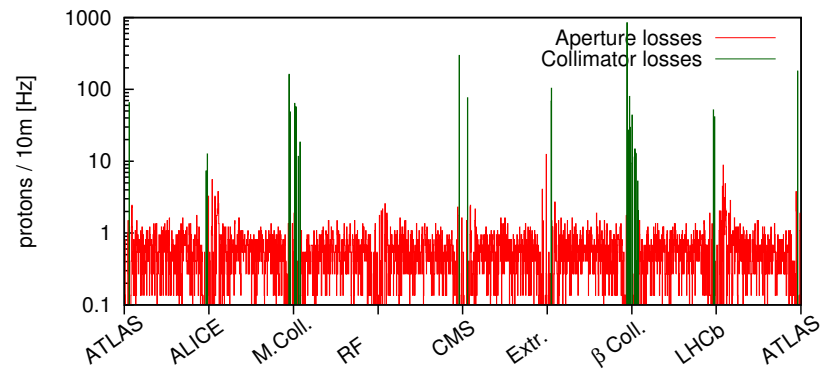

Fig. 3. The loss map produced from both inelastic and elastic collisions between protons in the beam and hydrogen atom at rest. This example shows the result of a simulation of $3.5 \mathrm{TeV}$ beam energy for beam 1, with a simulated pressure map. This is further explained in [13].

\section{ShOWER Formation in the Long Straight SECTIONS OF THE LHC}

The interaction of protons in the LHC beam with elements of the machine, collimators or residual gas molecules in the vacuum chamber results in a variety of processes and distribution of final state particles. The proton interactions in collimators generally result in inelastic processes and the formation of secondary particle showers composed of particles like pions and muons. For the case of showers induced in collimators around $\mathrm{LHCb}$, the relevant collimators for normal operation are tungsten tertiary collimators (TCTs) located $74 \mathrm{~m}$ and $118 \mathrm{~m}$ from the experimental interaction point. The interaction of a proton with a gas nucleus can be elastic or inelastic, producing quasi-elastic intact protons or initiating a secondary electromagnetic (EM) and hadronic particle shower. The collision of a high energy proton with a stationery atomic nucleus is a collision system strongly boosted in the forward direction and results in secondary particle showers peaked in the beam direction.

For the case of collimation-induced backgrounds, the EM and hadronic showers themselves are initiated in the tungsten insert block of the tertiary collimators. The LHC tracking calculation gives the distribution of inelastic proton-nucleus events in the tungsten block, and so the cascade is initiated with a proton-Tungsten nuclear interaction at the relevant location. This interaction itself can be calculated with an explicit DPMJET-III [14] calculation and an appropriate boost to the frame of the collimator, or implicitly in the FLUKA source routine. Both methods give the same results. For the case of beam-gas interactions, the vacuum pressure map measured by the LHC vacuum gauges in the straight region around the LHCb experiment [15] is sampled to distribute inelastic proton- $\mathrm{H}_{2}$ collision products. Each proton-nuclear event is simulated using DPMJET-III. The boosted reaction products are then showered towards the IP. Note that the proton-nucleus interactions produce many high energy forward pions, with the shower transverse size growing transversely as the particles move towards the experiment. An important topological difference between collimator-induced showers and beam-gas collision induced showers is produced when the dense collimator material moderates the shower development and reduces the energy of the forward pions. This results in a 
higher energy and more forward peaked shower from beamgas interactions than collimator interactions as can be observed in Fig. 4

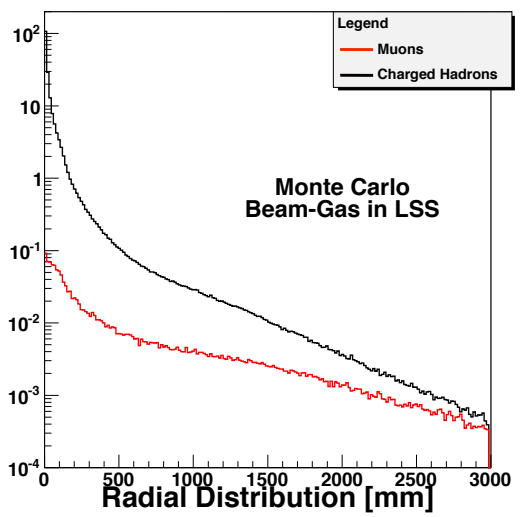

(a)

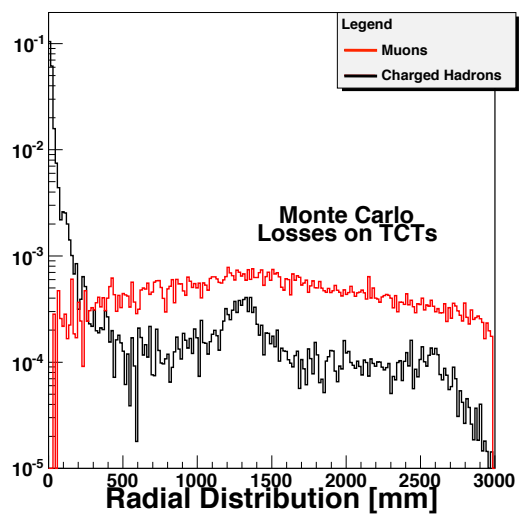

(b)

Fig. 4. Radial charged hadrons and muons distributions arriving at the $\mathrm{LHCb}$ cavern for beam 1 at $3.5 \mathrm{TeV}$, for beam gas in LSS on the top (a) and halo on TCTs on the bottom (b).

The measurement of the residual gas pressure profiles in the LHC by vacuum gauges is an essential input to beamgas calculations. The residual gas composition is a mix of species, dominated by $\mathrm{H}_{2}$ in cold regions and by $\mathrm{CH}_{4}$ in warm regions of the machine, but it includes other species such as $\mathrm{CO}_{2}$ and $\mathrm{CO}$, and a full pressure profile by species is needed for complete MIB calculations. However, the measurements are often formulated in terms of $\mathrm{H}_{2}$-equivalent species, where the pressure of non- $H_{2}$ species is replaced by an equivalent number of $\mathrm{H}_{2}$ molecules according to the ratio of the protonnucleus interaction cross section. This equivalent $H_{2}$ profile gives a feeling for total MIB rates but, due to differing interaction kinematics, does not fully describe the MIB produced from separated-species pressure profiles. In this work, $\mathrm{H}_{2}$ equivalent profiles are used, because they relate directly to static pressure measurements in the LHC. The potential impact of true species or equivalent $H_{2}$ pressure profiles on MIB rates and distributions is under study. However, to allow reweighting of secondary particle distortions, the location and parent atomic number of proton-nucleus collisions is recorded and passed down the secondary particle showers and stored in the secondary particle data arrays.

\section{A. The simulation model of the long straight sections close to $\mathrm{LHCb}$}

The hadronic and EM showers initiated by the protonnucleus inelastic interactions are calculated using the shower Monte Carlo FLUKA [17], [18]. A geometrical model of the LHC straight section around $\mathrm{LHCb}$ was constructed, comprising of tunnel, beam pipe, all accelerator elements and the tunnel shielding. This model is described in detail in [19] and summarised here.

The model is divided into separate pieces for the left side and the right side of the machine around the IP as viewed from the middle of the ring (LSS8-left and LSS8-right respectively) for computational simplicity and because of the presence of the LHCb detector at the IP. The model consists of the LSSs from $\pm 280 \mathrm{~m}$ from the IP to the interface plane between the machine and experiment at $-2.1 \mathrm{~m}$ for beam 1 (LSS8left) and $+19.9 \mathrm{~m}$ for beam 2 (LSS8-right). Taken together, the two halves of the model provide a complete picture of LSS8, with a gap in the middle for the detector. The LHC layout database [20] and optics web-page [21] was used to produce the layout, and engineering drawings were taken from the CERN drawing database [22].

The description of the final triplet magnets were adapted from the existing model for LSS1 [23]. The magnetic field in the final focusing triplet is modeled with an explicit field map in the quadrupoles (known as Q1, Q2 and Q3). The model for the normal conducting separating dipole (D1) was adapted from the superconducting separating dipole (D2) in the LSS1 FLUKA model [23] already mentioned, and the remaining quadrupoles are models of the standard LHC components [23]. In the separation dipoles and the remaining quadrupoles the fields are ideal and localized to the vacuum chamber. For LSS8, new models were made of the corrector magnets around the experiment, which are part of the $\mathrm{LHCb}$ inner crossing angle bump. The fields for these elements are ideal. The reference orbit for an incoming and outgoing nominal proton was checked against the LHC reference optics and agreement achieved.

The collimators in the LSS of IR8 are the tertiary halo collimators (TCTs) for the circulating beam. These are designed to intercept the tertiary beam halo before it hits the superconducting final triplet magnets and also provide protection in the event of anomalous beam dump events. They are located on the incoming beams, with one collimator with vertical jaws and one with horizontal jaws, with a tungsten jaw inset for proton collimation. The horizontally collimating TCTH is derived from a standard LHC one-beam collimator [23], [24] and is located around $118 \mathrm{~m}$ from the IP. The TCTVB is a two-beam vertical collimator located around $74 \mathrm{~m}$ from the $\mathrm{LHCb}$ interaction point on the incoming beam. The close proximity of the two beams at the TCTVB location means the collimator is a two-beam type, consisting of a large aperture to accommodate both beams and a shaped collimator block with a tungsten insert, designed to ensure only the incoming beam impacts the tungsten. The model of the TCTVB is a specific two-beam collimator model, adapted from the onebeam standard LHC collimator. The collimators are aligned 
with the incoming beams, and the jaw position set by the optics and beam energy.

The LHCb interaction region is close to the LHC injection of beam 2 and therefore injection-related elements are in the model of the LSS around LHCb.

Beam 2 is injected in the right-side of the IP from the outside of the accelerator ring, as shown in Fig. 1. Septum magnets (MSI) deflect the beam in the horizontal plane by $12 \mathrm{mrad}$ onto the horizontal orbit. The MSI also has an aperture for the circulating beam 1 , and a new model of the elements was introduced. The beam orbit is kicked horizontally by the four kicker modules of the MKI, the injection kicker. The kicker is currently described in a simple way. The LSS8-right model also contains injection-related collimators for injection protection. For further details on the description of these elements see [19].

The tunnel shielding of LHCb is modelled in terms of blocks of iron and concrete in the model of the LHC tunnels. The role of the shielding is to screen the LHCb detector from the MIB particle fluxes, and is expected to be most effective for the charged hadron contribution. For example, on the left-hand side of the IP (RB84), the shielding is grouped in three regions, $80 \mathrm{~cm}$ of concrete closest to the IP, $80 \mathrm{~cm}$ of iron and $120 \mathrm{~cm}$ of concrete in the tunnel, and then $80 \mathrm{~cm}$ of iron and $120 \mathrm{~cm}$ of concrete forming a tunnel chicane.

An overview of the FLUKA geometrical model of LSS-left as it has been implemented is shown in Fig. 5. In this paper, $z$ is defined as the direction along the beam.

The EM and hadronic showers were propagated with a 20 $\mathrm{MeV}$ cut on kinetic energy of all charged hadrons, electromagnetic particles and muons, whilst allowing neutrons down to thermal energies. The multiplicity of the EM cascade was controlled with a leading particle bias on the cascade below 1 $\mathrm{GeV}$. The choice of cut or bias was checked not to impact the LHCb background fluxes scored above $20 \mathrm{MeV}$. The secondary particle products from the showers are scored at the interface of the tunnel with the LHCb cavern [25]. An exchange file format has been defined which contains all the information needed in order to import the particles into the next step of the simulation, that of the LHCb detector. All particle species are recorded with energy, weight (to account for the use of biasing in the cascades), phase space coordinates, arrival time (with $\mathrm{t}=0$ corresponding to the original beam proton interaction and with respect to the reference particle), the longitudinal position of the original loss location and the species of the parent interaction atom.

\section{Simulation of Machine Induced Background PARTICLES IN THE LHCB DETECTOR}

In order to ascertain the impact of various MIB sources on $\mathrm{LHCb}$, these particles must be imported and simulated in the standard software of the LHCb experiment.

The simulation application Gauss [26], [27] mimics what happens in the detector. Like all LHCb data processing software it is based on a framework named Gaudi [28], [29] and as such Gauss follows its architectural design, with a clear separation between data and algorithms and with well defined

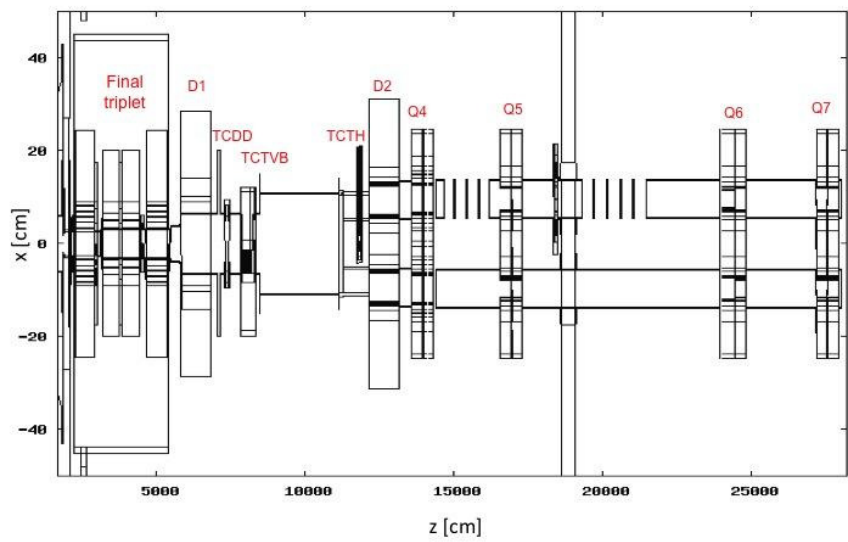

Fig. 5. Layout of the long straight section left of $\mathrm{LHCb}$ as used in the simulation. The IP is at $z=0$.

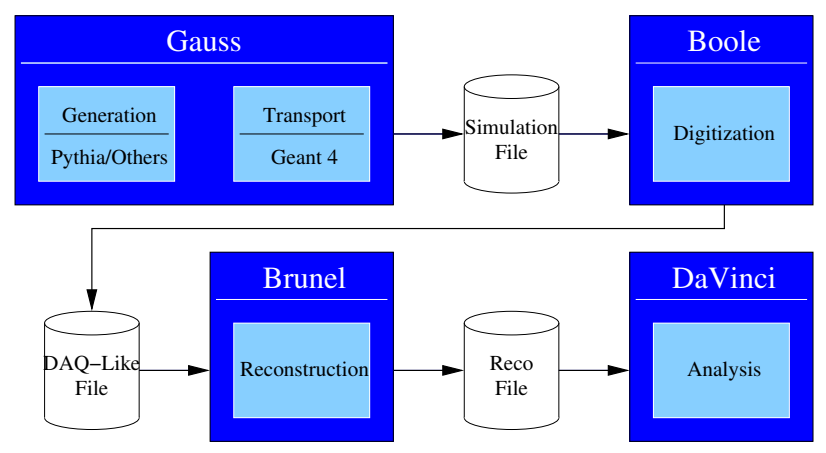

Fig. 6. The LHCb data simulation chain. Particle generation and interactions with the detector are simulated in Gauss. Boole translates the particle hits on the sensors into a DAQ-equivalent format. Brunel reconstructs tracks and particles from the DAQ information while analysis is performed using DaVinci.

component interfaces. The Gauss simulation specific code is encapsulated in dedicated Algorithms and Tools (smaller pieces of code) callable and controllable from the framework.

Gauss is divided into two phases, generation of the initial particles and simulation of their transport through the $\mathrm{LHCb}$ detector making use of the Geant4 toolkit [30]. For the generation phase various generator algorithms exist, each dedicated to represent a specific particle source. Most notably various type of events, for example specific physics signals or softQCD events produced in proton-proton collisions at the IP, are generated using libraries developed in the HEP community (e.g. PYTHIA [31]). Following this scheme a dedicated MIB algorithm has been implemented, able to import and sample the MIB estimates.

As indicated in Fig. 6, after the simulated data has been generated and transported through the LHCb detector description by Gauss, the data is processed by Boole. This application calculates the expected detector response and transforms the data into a DAQ equivalent format. This, as well as real data, is reconstructed into standard track and particle candidates in Brunel. Finally data analysis is predominantly performed using the DaVinci application [26]. 
TABLE IV

MIB ESTIMATE FILE FORMAT. EACH PARTICLES IS REPRESENTED THROUGH A LINE CONTAINING ALL THE VARIABLES

\begin{tabular}{ll}
\hline Element & Description \\
\hline LossID & Proton loss identifier \\
LossT0 & LHC clock offset of proton loss (ns) \\
LossZ & Proton loss z coordinate (mm) \\
LossA & Type of atom involved in proton loss \\
LossW & Proton loss "weight" (likelihood) \\
PartPdgId & PDG particle ID number \\
PartX & Particle x coordinate at interface plane $(\mathrm{mm})$ \\
PartY & Particle y coordinate at interface plane $(\mathrm{mm})$ \\
PartZ & Particle z coordinate origin at creation $(\mathrm{mm})$ \\
PartDx & Particle x direction cosine at interface plane \\
PartDy & Particle y direction cosine at interface plane \\
PartEk & Particle kinetic energy (GeV) \\
PartDt & LHC clock offset for particle arrival at interface plane (ns) \\
PartW & Particle "weight" (likelihood) \\
\hline
\end{tabular}

\section{A. File exchange format}

In order to simplify the task of importing MIB estimates into the LHCb simulation system it is essential that the data is presented in a consistent way. A text based file format has been created in order to provide a well defined interface for the exchange of particle information. In addition to assuring consistency between the various background sources it simplifies the analysis process as standardised tools can be created.

Whenever possible a file represents only one background type. For example in the case of beam-gas interactions, having one file for each type of gas atom simplifies the task of rescaling the sources with different gas densities. Furthermore, this allows them to be studied separately as well as to combine them with appropriate scaling factors in the simulation stage.

The particle information of the estimate files is given at a vertical interface plane at a certain distance from the $\mathrm{LHCb}$ IP. In order to make sure all relevant detector components are inside the simulated area, the preferred interface planes are at $z=-2.1 \mathrm{~m}$ for beam 1 and at $z=19.9 \mathrm{~m}$ for beam 2 , where $z=0$ is the IP location.

The estimate files are flat text files where the particle entries are separated by line-breaks. Each line represents a particle where the various values are space-separated, representing the variables listed in Table IV.

As can be seen from the Table, there are two main type of properties, those related to secondary particles and those related to the original proton loss. The loss information is related to the proton interaction from which the particle has originated. Thus several particles can contain the same loss information. In effect the MIB event originates from a proton loss and contains the particles associated to this loss through the LossID element. The reason for this system is to keep the correlations between the individual particles, so that particles that are members of the same shower can be re-generated as such.

The variable LossW is a special weighting parameter requiring further explanation. The value of this variable is a relative likelihood of the associated MIB event with respect to the sum of the weights of all $N$ events in the estimate file. The probability $P$ of choosing a certain event from the estimate is given by equation (2).

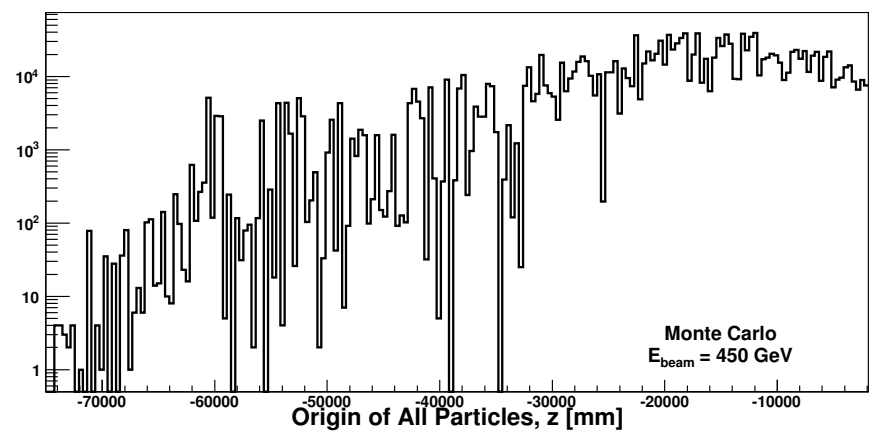

Fig. 7. Distance to IP of primary proton loss for beam-gas in the LSS at $450 \mathrm{GeV}$ for beam 1. Vertical axis indicate particle flux from this position.

$$
P=\frac{\operatorname{Loss} \mathrm{W}}{\sum_{0}^{N} \operatorname{Loss} \mathrm{W}}
$$

Similarly the PartW parameter is the probability that the particle will be generated when the associated event is selected and is related to the probability of the particle reaching or not the interface plane due to interactions with the material traversed while being transported from the initial loss.

The rest of the elements are used either for scaling purposes or, in the case of the particles kinematic variables, for continued propagation of the particles.

As the estimate files are potentially very large, it is not possible to contain all information in memory. However, fast and easy access to the data is needed as random sampling procedures are utilized in the generation algorithms. For this purpose the reading and parsing of flat text files is too time consuming and a binary file format is needed. For this purpose the ROOT [32] software is used. This is convenient as the LHCb software suite is already ROOT-compatible and the resulting data format is both platform and architecture independent. The event and particle data are saved in two separate tuples (or specifically TTrees). The association between the two is preserved through the use of reference indices.

The translation from the flat estimate files to the ROOT file format is done through a python script. The script also allows for the addition of header information. This header contains global information relevant for the re-generation of the particles, ranging from estimate scaling to beam direction and interface plane position.

The ROOT file can be analyzed directly, which already allows for an in-depth understanding of the MIB sources. As an example Fig. 7 shows the distance from the IP of the primary proton loss for beam-gas in the LSS at $450 \mathrm{GeV}$. Fig. 4(a) and 4(b) have also been produced by analyzing the file for $3.5 \mathrm{TeV}$.

\section{B. MIB generator algorithm}

The MIB algorithm is designed to produce MIB events in the generator phase of the Gauss application and pass them to the subsequent processing simulation phase. This algorithm can run stand-alone or in conjunction with other particle generators to create a mixed sample. In order to enable several MIB sources to be evaluated in the same event, the actual 
particle sampling is not performed in the algorithm itself, but rather delegated to a set of generator tools. Each tool instance is set up to represent a given MIB source defined by its settings and the ROOT file to which it has access. The software can be controlled through user-configurable options, giving information about, amongst others, which source, rate and production location should be used.

Whenever Gauss calls the MIB generator algorithm, the call is passed on to the MIB tools instances. In each of them the number of MIB events created are randomly determined in a way consistent with the related MIB source. These events are randomly selected from the associated estimate ROOT file. In order to take the weight, and thus the probability, of the events into account one can not simply randomly select the events. For this the LossW parameter must be used.

Each event $i$ gets a parameter $s_{i}$ assigned which is equal to the sum of the LossW parameter for the current event and all events listed before it $\left(s_{i}=\sum_{0}^{i} \operatorname{Loss} W\right)$. For the event selection, a uniformly distributed random number in the range $\left[0, \sum_{0}^{N} \operatorname{Loss} \mathrm{W}\right]$ is attained. The event where $s_{i}$ is larger than or equal to the random number and that of the preceding event was smaller is the event to be selected.

In order to reduce the amount of file access requests required during the event search, every $J$ th event (where $J$ is a usersettable parameter) is stored in memory. The search is first conducted on these envelopes in order to find the approximate location of the event in the file. The search directly in the file is thus limited to a subset of $J$ events, saving computing time.

When the events have been selected, the associated particles are generated with a probability equal to the parameter PartW. The particles are created using the particle ID and kinematic parameters. The MIB algorithm combines the particles and events (if any) arriving from the MIB tools, and passes them over to the simulation step of Gauss in order to ascertain their impact on the $\mathrm{LHCb}$ experiment. Particles from other generators can also be added at this point. In particular MIB events can be generated together with standard proton-proton interactions, providing a complete view of the environment in which the $\mathrm{LHCb}$ detector collects data.

\section{EXAMPLE SIMULATION RESULTS}

By utilizing the applications and algorithms described in the previous sections, MIB simulation files are produced. These can be compared to the simulations of proton-proton interactions as well as real data in order to ascertain the viability of our estimates and ultimately to explain various characteristics of the data. When a populated bunch from one beam crosses an empty bunch from the other, no protonproton interaction is possible, thus the resulting signal only contains MIB and beam-gas from within the experiment. This is referred to as a beam-empty (BE) event as opposed to a beam-beam (BB) event, where both bunches are filled. Strictly, one should note that BE events can contain a small amount of actual proton-proton collisions, from debunched beam or mismatch with the injectors, which to some extent will fill the empty bunch locations. This is particularly true for empty bunch locations close to filled ones. This contribution

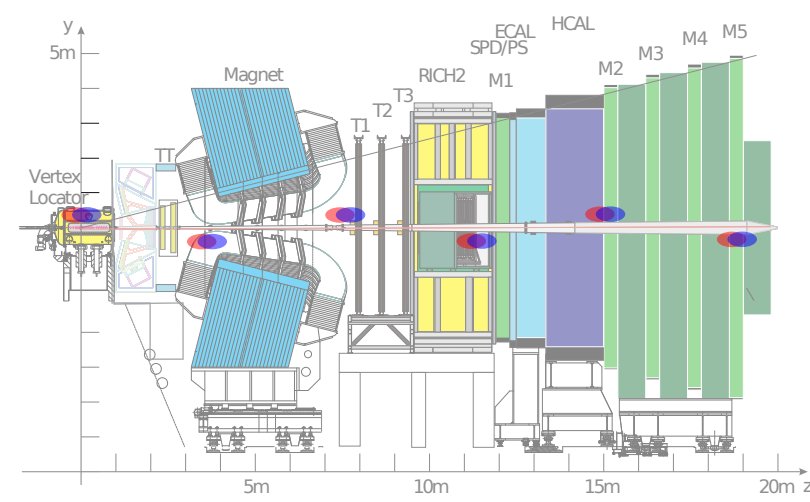

Fig. 8. Bunch crossing locations within the LHCb. The red and blue dots represent the proton bunches. Two time slots are shown; during crossing at IP (upper dots) and offset by $12.5 \mathrm{~ns}$ (lower dots).

is neglected in this paper as BE events can be selected with a small contamination.

A good understanding of the MIB signal characteristics is needed to discriminate and understand its contributions. This section shows a few examples of MIB characteristics as obtained with the simulation chain described and indicating what criteria can be used in order to separate the various components.

Machine induced background typically arrives on time with the originating proton bunch, making it hard to distinguish between the MIB particles and the products of the protonproton interactions at the IP. However this is only true for particles traveling away from the IP. As the bunch travels towards the IP it is only accompanied by MIB.

The LHC has a nominal bunch separation of $25 \mathrm{~ns}$, corresponding to about $7.5 \mathrm{~m}$. This means that the bunches meet each other at every $3.75 \mathrm{~m}$ as can be seen from figure 8 . When MIB from the two beams arrive at these locations they are undistinguishable from each other. In other regions between these locations the timing of the detectors signals can allow the discrimination of the MIB originating from the two beams.

An example of such a region is shown in Fig. 9 for one of the trackers located at about $9.5 \mathrm{~m}$ from the IP. One can clearly see that the signal from beam 1 MIB is on-time with the proton-proton signal, while beam 2 MIB is separated by about $10 \mathrm{~ns}$. Due to the single arm design of LHCb the visibility of beam 2 MIB by the spectrometer is highly reduced. This is a result of both the geometry of the detector and the read out of the electronics. The data acquisition has been designed to record particles as they travel through the detector, originating from the region of the IP. In order to allocate the various subdetector hits to the same interaction event, timing information is used. MIB from beam 2 arrives at the detector elements in the wrong order and would thus be assigned to different events, making the signal in the sub-detectors appear as uncorrelated.

MIB analysis using single sub-detectors is possible for both beam directions when appropriate read out timing is used. This can be used for example by special background sensors called the Beam Loss Scintillators, located upstream of the experiment and with readout independent of the data acquisition, 


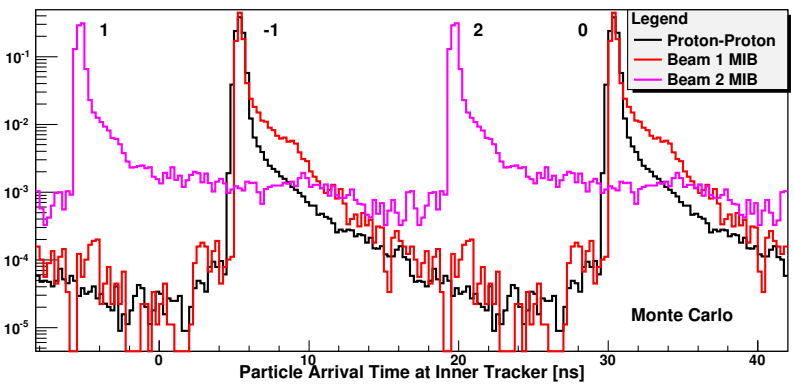

Fig. 9. Time of arrival of particles at the $\mathrm{LHCb}$ inner tracker originating from MIB from beam 1 and 2 as well as proton-proton interactions. The scaling is arbitrary. Bunch crossing ID is indicated at each peak.

allowing monitoring of background in all operations condtions.

Another feature of MIB events that may allow their discrimination from other type of events is particle multiplicity, that is reflected in the number of signal channels per event in the different sub-detectors. An example of this can be seen in Fig. 10(a), where for $450 \mathrm{GeV}$ beam energy the MIB events have a higher cluster multiplicity distribution than protonproton collision in the Vertex Detector allowing for a clear separation between this two different categories of events. It can be argued that the higher multiplicity component is due to the fact that the MIB particle showers have had a longer distance to develop compared to the collisions. However, at higher energies as shown in Fig. 10(b)) for $3.5 \mathrm{TeV}$ beam energy, this effect is much less pronounced and multiplicity distributions are not as useful to distinguish between MIB events and proton-proton collisions. In addition the rate of MIB events in BB events is expected to be so low with respect to proton-proton collisions in normal operating conditions that it is more efficient to investigate MIB's discriminating variables with respect to local beam gas in the Vertex Detector in $\mathrm{BE}$ events.

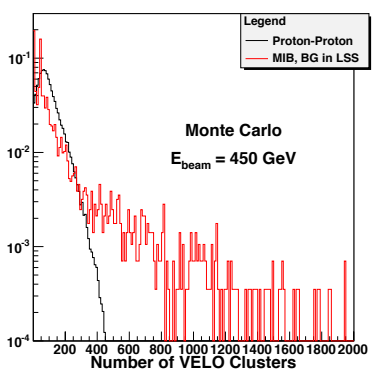

(a)

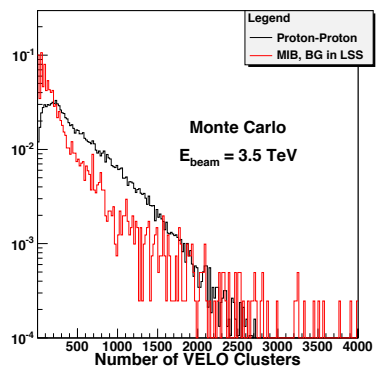

(b)
Fig. 10. Total cluster multiplicity in the vertex detector due to proton-proton collisions and MIB from beam-gas in the LSS at $450 \mathrm{GeV}$ (a) and $3.5 \mathrm{TeV}$ beam energy (b). The curves have each been normalized to 1 .

Charged particle traversing the detector can be reconstructed as tracks by the Brunel application. As the MIB events arrive from outside of the experiment one can expect the related tracks to have a comparatively flat slope. This track slope parameter is defined as the distance traveled in the plane perpendicular to the beam axis per unit length along the same axis. Figure 11 shows the slope of charged MIB particles as they are given at the interface plane upstream

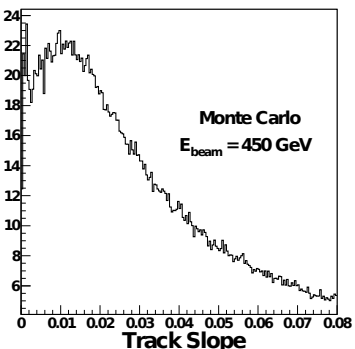

Fig. 11. Slope of charged particles for beam-gas in the LSS at $450 \mathrm{GeV}$ as given at the interface plane $2.1 \mathrm{~m}$ upstream from the IP.

of the experiment. This is reflected in slope of the tracks as reconstructed from MIB events that can be seen together with those from beam gas occurring locally in the Vertex Detector in Fig. 12(a)

Another discriminating criteria that has been investigated is the radial distance from the track to the nominal interaction point (IP). Particle originating in the area of the Vertex Detector should have a small radial distance to the IP while MIB events typically have a higher value for this variable. This criteria, together with the track slope, may enable the distinction between MIB and beam-gas interactions in the experiment itself, as can be seen from Fig. 12.

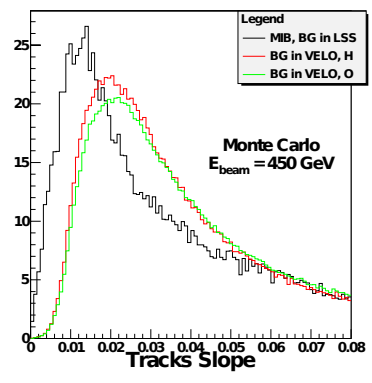

(a)

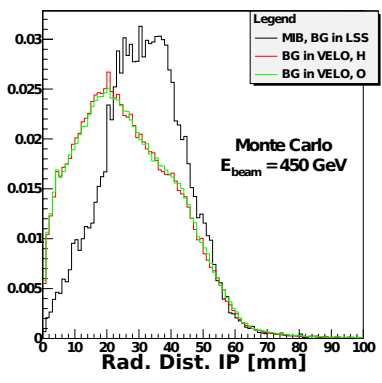

(b)
Fig. 12. Track slope (left) and radial distance to the IP (right) for beam-gas in the LSS and in the vertex locator at $450 \mathrm{GeV}$.

By comparing a linear combination of these two distributions to the signal recorded from $\mathrm{BE}$ events, the fraction between the two can be approximated. Furthermore, cuts on these variables can be used to increase the purity of MIB events either at the trigger level for dedicated background studies or during analysis. At $450 \mathrm{GeV}$, the loose machine settings limits the number of MIB sources to the beam-gas in LSS. At higher energies the tighter collimator settings mean background sources from the TCT collimators become larger and need to be folded into the analysis, making their analysis more complex.

\section{CONCLUSIONS}

Machine induced background at the LHC arises from proton interactions with elements of the machine and gives a flux of background particles into the $\mathrm{LHCb}$ experiment. It is important to evaluate the amount and characteristics of this background, not only for the design of an experiment but also 
during operation in order to understand possible degradation of the conditions and be able to take corrective measures. The backgrounds arise in the machine from proton interactions with beam gas molecules, either close to or far away from the experiment, interactions of protons with the beam collimation system and from fluxes produced by beam-beam collisions in the other LHC experiments. Any calculation scheme for these background sources needs to simulate both the machine and detector response, and would be a valuable tool for understanding and mitigating the impact on the experiment and possibly providing information for future experimental upgrades.

In this paper a full and novel chain of simulations as developed for the $\mathrm{LHCb}$ experiment has been described, and a coherent data flow designed. A modular architecture allows to study all sources independently from each other, as well as combine them for a complete and comprehensive understanding of the background in the experiment in different modes of operation of the machine. Furthermore, steps in the simulation chain can be modified without impacting the overall data flow and analysis. The resulting simulation chain and data flow uniquely models the LHC machine and detector components, allowing cradle-to-grave analysis, with well-defined interfaces between the simulation steps. The comprehensive simulation chain begins with models of the LHC machine, covering the beam-gas interactions and the collimation system and continues with a model of the LHCb detector. MIB simulated data can then proceed through event reconstruction and selection. Validation of the methodology and data flow has been performed and the system has allowed to quickly compare measured data at $450 \mathrm{GeV}$ showing qualitative agreement of particle flux distributions in the LHCb detector. While not all of the MIB sources have been considered in this paper, for example the background arising from momentum halo formation, the methodology is applicable to any MIB source in the LHC.

The scope of this paper was a full presentation and validation of the complex simulation chain and data flow. Studies are in progress for a beam energy of $3.5 \mathrm{TeV}$, exploiting the simulation chain, to analyse the data and compare them to the various sources at the different energies collected. Once good quantitative agreement has been proved, an in depth analysis of expected background conditions for the nominal machine will be carried out.

\section{ACKNOWLEDGMENT}

The authors would like to acknowledge the LHC collimator group for providing the loss rates for the cleaning and the Vacuum group for the residual pressure simulations and measurements. The authors also wish to acknowledge all contributors to the offline software of the $\mathrm{LHCb}$ experiment, on which the last steps of the MIB simulation chain is based on.

\section{REFERENCES}

[1] L. Evans et al, "LHC Machine," 2008 JINST 3 S08001, Aug. 2008

[2] The LHCb Collaboration, "The LHCb Detector at the LHC," 2008 JINST 3 S08005, Aug. 2008
[3] R. W. Assmann et al, "The final collimation system for the LHC," $L H C$ PROJECT-Report-919, Jul. 2006

[4] Chiara Bracco, "Commissioning Scenarios and Tests for the LHC Collimation system," Ph. D. Thesis

[5] G. Ripken and F. Schmidt, "A symplectic six-dimensional thin-lens formalism for tracking," CERN-SL-95-12. CERN-SL-95-12-AP. DESY-95063, Apr 1995. http://cdsweb.cern.ch/record/281283

[6] K. Heinemann, G. Ripken and F. Schmidt, "Construction of Nonlinear Symplectic Six-Dimensional Thin-Lens Maps by Exponentiation," DESY95-189, 1995. http://arxiv.org/pdf/acc-phys/9510005

[7] G. Robert-Démolaize, "Design and Performance Optimization of the LHC Collimation System," http://cdsweb.cern.ch/record/1004869

[8] G. Robert-Démolaize, R. W. Assmann, S. Redaelli and F. Schmidt, "A New Version of SixTrack with Collimation and Aperture Interface," Proceedings of the Particle Accelerator Conference, 2005. PAC 2005. pp. 4084-4086, May 2005. http://cdsweb.cern.ch/record/851591

[9] T. Trenkler and J. B, Jeanneret, "K2, A software package evaluating collimation systems in circular colliders (manual)," SL-Note-94-105-AP, Dec. 1994. http://cdsweb.cern.ch/record/703539

[10] R.B. Appleby et al, "Simulations of the LHC Collimation System," The proceedings of IPAC10, Kyoto, Japan, May 23-28 2010

[11] O. S. Brüning, et al, "LHC Design Report," CERN, 2004, http://cdsweb.cern.ch/record/782076

[12] Y. Inntjore Levinsen, H. Burkhardt and V. Talanov, "Simulation of Beam-Gas Scattering in the LHC," PAC Vancouver, May 2009, submitted for publication.

[13] Y. Inntjore Levinsen, R. B. Appleby and H. Burkhardt, "Beam-gas Loss Rates in the LHC," IPAC Kyoto, Jun. 2009, http://cdsweb.cern.ch/record/1269890

[14] S. Roesler, R. Engel and J. Ranft, "The Monte Carlo event generator DPMJET-III," arXiv: hep-ph/0012252, HEP-PH/0012252. http://cdsweb.cern.ch/record/481840

[15] The LHC vacuum group, private communication.

[16] A. Rossi, "Estimations for beam-gas scattering calculations for the first year run," Presented at LHC Background Study Group. http://indico.cern.ch/conferenceDisplay.py? confId $=54698$

[17] A. Fasso; A. Ferrari, J. Ranft and P. R. Sala, "FLUKA: A multi-particle transport code," CERN-2005-10 (2005), INFN/TC_05/11, SLAC-R-773

[18] G. Battistoni, et al., "The FLUKA code: Description and benchmarking," Proceedings of the Hadronic Shower Simulation Workshop 2006, Fermilab 6-8 Sep 2006, M.Albrow, R.Raja eds., AIP Conference Proceeding 896, 31-49, (2007)

[19] R. B. Appleby and A. Mereghetti, "The FLUKA Model of IR8," CERNLHC-Project-Note-427, http://cdsweb.cern.ch/record/1238789

[20] "LHC Layout Database," https://cern.ch/ts-dep-ic/LI/

[21] "LHC optics web page," http://cern.ch/lhcoptics

[22] “CERN Drawing Database," http://cern.ch/edms-service/CDD/

[23] C. Hoa, F. Cerutti, E. Wildne, "Energy Deposition in the LHC Insertion Regions IR1 and IR5," LHC Project Report 1167

[24] M. Magistris, M. Santana Leitner, K. Tsoulou, M. Brugger, F. Cerutti, A. Ferrari and V. Vlachoudis, "Technical Description of the implementation of IR7 section at LHC with the FLUKA transport code," CERN-AB-Note031-ATB

[25] G. Corti, M. Lieng and R. Jacobsson, "Requirement for Machine Induced Backgrounds Studies in LHCb," LHCb-INT-2009-017

[26] The LHCb Collaboration, R. Antunes Nobrega et al., "LHCb Computing Technical Design Report, CERN-LHCC-2005-019 (2005).

[27] S. Miglioranzi et al, "The LHCb Simulation Application, Gauss: Design, Evolution and Experience" Submitted to Proceedings of Conference on Computing in High-Energy and Nuclear Physics 2010, Taipei, Taiwan, 18-22 Oct 2010, CERN-LHCb-PROC-2011-006.

[28] G. Barrand et al., "GAUDI - A software architecture and framework for building HEP data processing applications, Comput. Phys. Commun. 140 (2001) 45.

[29] M. Clemencic, H. Degaudenzi, P. Mato, S. Binet, W. Lavrijsen, C. Leggett and I. Belyaev, "Recent Developments In The LHCb Software Framework GAUDI, J. Phys. Conf. Ser. 219 (2010) 042006.

[30] J. Allison et al, "Geant4 developments and applications," IEEE Transactions on Nuclear Science. v. 53, n. 1, p. 270-278, Feb. 2006.

[31] T. Sjostrand, S. Mrenna and P. Z. Skands, "PYTHIA 6.4 Physics and Manual", JHEP 0605, 026 (2006).

[32] R. Brun and F. Rademakers, "ROOT - An Object Oriented Data Analysis Framework", Proceedings AIHENP96 Workshop, Lausanne, Sep. 1996, Nucl. Inst. and Meth. in Phys. Res. A 389 (1997) 81-86. See also http://root.cern.ch/ 